\title{
Metastatic Colorectal Cancer Treated with Herbal Pharmacopuncture during FOLFIRI Chemotherapy: A Case Report
}

\author{
Jung-Woo Lee Jae-bok Han $^{\mathrm{b}}$ Sung-su Kim ${ }^{\mathrm{b}}$ Shin Seong ${ }^{\mathrm{b}}$ \\ ${ }^{\mathrm{a}}$ Department of East-West Medicine, Graduate School, Kyung Hee University, and \\ ${ }^{b}$ Soram Korean Medicine Hospital, Seoul, South Korea
}

\section{Key Words}

FOLFIRI chemotherapy $\cdot$ Herbal pharmacopuncture $\cdot$ Korean medicine $\cdot$ Metastatic colorectal cancer

\begin{abstract}
According to the 2008 World Cancer Report by the World Health Organization (WHO), colorectal cancer is one of the leading cancers worldwide. This case study evaluates the effectiveness of Korean medicine treatment, including herbal pharmacopuncture (HP), as a complementary treatment during FOLFIRI chemotherapy. A 73-year-old male who was diagnosed with metastatic colorectal cancer (mCRC) in September 2012 was treated concurrently with HP and FOLFIRI chemotherapy for 4 months. The effectiveness of the combined therapy was evaluated by CT. Furthermore, quality of life was assessed using a visual analogue scale. The tumor mass sizes of lung and lymph node metastases decreased, and the side effects caused by chemotherapy were mitigated. Based on these results, this case report suggests that Korean medicine treatment including HP therapy could be a complementary therapy for $\mathrm{MCRC}$.

(c) 2014 S. Karger AG, Basel
\end{abstract}

\section{Introduction}

Colorectal cancer refers to a malignant tumor in the rectum or colon. Most colorectal cancers are adenocarcinomas, which occur in the mucous membrane of the colorectal area [1]. It is known that colorectal cancer originates from an adenomatous polyp, which is a 
benign tumor. Other rare primary forms of colorectal cancer include neuroendocrine, squamous cell, adenosquamous, spindle cell, and undifferentiated carcinomas [2].

According to the 2008 World Cancer Report by the World Health Organization (WHO), colorectal cancer accounts for $9.4 \%$ of all cancers worldwide. This means that there are 1 million newly diagnosed colorectal cancer patients every year. For males, colorectal cancer is the fourth most common cancer following lung, prostate, and gastric carcinomas. For females, it is the third most common cancer following breast and uterine cervical carcinomas [3].

Especially for metastatic colorectal cancer (mCRC), the complete response rate for conventional chemotherapy is very low. According to Ferrarotto et al. [4], the 5-year survival rate is $10.8 \%$, and only $2.2 \%$ of mCRC patients show a complete response to chemotherapy. Moreover, for mCRC patients, the treatment differs according to whether or not surgery is possible. If surgery is not a treatment option, chemotherapy is used for palliative treatment.

\section{Case Report}

A 73-year-old male patient diagnosed with mCRC was admitted to our hospital in September 2012. He had been diagnosed with colorectal cancer in March 2005 and had been treated with surgery and chemotherapy at that time. In September 2012, the patient was diagnosed with colorectal cancer with multiple metastatic nodules in the right and left lungs and multiple metastatic lymph nodes in both the supraclavicular area and the mediastinum. After the diagnosis, he was treated with FOLFIRI chemotherapy along with Panax ginseng and Cordyceps sinensis herbal pharmacopuncture (HP) treatment. FOLFIRI chemotherapy is a commonly used cocktail combination of three chemotherapeutic agents: fluorouracil, leucovorin, and irinotecan. Immediately after starting chemotherapy, the patient was admitted to our hospital to be treated with HP therapy. When he first presented to our hospital, he had some side effects caused by FOLFIRI chemotherapy such as fatigue, stomatitis, nausea, and headache.

The patient was treated with FOLFIRI chemotherapy eight times every 2 weeks, with intravenous HP treatment three times per week. Several other forms of Korean medicine, including pharmacopuncture, acupuncture, moxibustion, and oral herbal medicines, were also applied as symptomatic therapies. Specifically, the side effects caused by FOLFIRI chemotherapy were treated with oral herbal medicine, and the symptoms were mitigated. The decrease of symptoms caused by chemotherapy was assessed using a visual analogue scale (VAS). However, after each chemotherapy treatment, some side effects that had diminished after oral intake of herbal medicine recurred. The oral herbal medicine used for nausea was Yi-jin-tang (二陳湯), for headache Cheong-sang-gyeon-tong-tang (淸上噣痛湯), and for stomatitis Hoei-choon-yang-gyeok-san (回春凉腷散). The VAS evaluations of the symptoms caused by chemotherapy are summarized in table 1 .

CT images were obtained three times during the course of treatment: in September 2012, October 2012, and finally in January 2013. The tumor sizes were found to have decreased at each CT imaging session. Compared to his first CT image in September 2012, the one in January 2013 showed significant decreases in both the pulmonary and lymph node areas. The radiological findings are shown in fig. 1. 
Lee et al.: Metastatic Colorectal Cancer Treated with Herbal Pharmacopuncture during FOLFIRI Chemotherapy: A Case Report

\section{Discussion}

Ginseng (Panax ginseng C.A. Meyer) has been used widely for thousands of years in traditional Eastern medicine to invigorate and vitalize physical functions. Also, it is claimed to be effective in combating stress, fatigue, oxidants, diabetes mellitus, and cancer [5]. Moreover, a randomized, placebo-controlled clinical trial of Panax ginseng has shown its antioxidant effects [6]. In addition, another study concluded that Panax ginseng eases the heart rate, increases lipolysis, reduces plasma lactate concentration, and maintains a good health by reducing stress [7].

Cordyceps sinensis has been used in traditional Eastern medicine for respiratory symptoms such as cough, sputum, and bronchial obstruction in asthma, tuberculosis, chronic bronchitis, and other diseases [8]. Therefore, we used this HP for treating the patient's metastatic pulmonary tumor. Also, studies have reported antioxidant [9] and anti-tumor [10] effects of Cordyceps sinensis.

Pharmacopuncture is a new form of therapy derived from a combination of herbal medicine and acupuncture. Herbal extracts are injected in the affected areas or intravenously to induce a therapeutic response [11]. The manufacturing process of the HP materials has been described previously [12]. HP, which is produced through a distillation process, is known to contain various flavonoids [13]. Flavonoids, as a group of compounds, are presumed to interfere with inflammation and carcinogenesis in various ways [14]. An in vivo carcinogenesis test showed inhibitory effects of flavonoids on skin tumor promotion [15]. A case report on the anticancer effect of HP on various kinds of cancers has been published [13]. In the case of cancers, HP could be more effective when injected intravenously than when injected subcutaneously at an acupoint. In our patient, by combining chemotherapy with Korean medicine therapy, not only did the tumor size decrease but the side effects caused by chemotherapy were also mitigated. HP therapy could be a complementary treatment with chemotherapy for mCRC patients. It is difficult to reach any conclusions about the efficacy of HP in mCRC from the present case only, but our findings suggest that complementary HP treatment with conventional FOLFIRI chemotherapy could be an alternative therapy for mCRC patients. In addition, more rigorous studies on the effectiveness of HP therapy are warranted.

\section{References}

1 Hamilton SR, Bosman FT, Boffetta P: Carcinoma of the colon and rectum; in Bosman FT, Carneiro F, Hruban RH, Theise ND (eds): WHO Classification of Tumours of the Digestive System. Lyon, IARC Press, 2010, pp 134-146.

2 Fleming M, Ravula S, Tatishchev S, Wang H: Colorectal carcinoma: pathologic aspects. J Gastrointest Oncol 2012;3:153-173.

3 World Health Organization: World Cancer Report, 2008.

-4 Ferrarotto R, Pathak P, Maru D, Agarwal A, Overman M, Hoff PM, Kopetz S: Durable complete responses in metastatic colorectal cancer treated with chemotherapy alone. Clin Colorectal Cancer 2011;10:178-182.

-5 Yue PY, Mak NK, Cheng YK, Leung KW, Ng TB, Fan DT, Yeung HW, Wong RN: Pharmacogenomics and the Yin/Yang actions of ginseng: anti-tumor, angiomodulating and steroid-like activities of ginsenosides. Chin Med 2007;2:1-21.

6 Kim HG, Yoo SR, Park HJ, Lee NH, Shin JW, Sathyanath R, Cho JH, Son CG: Antioxidant effects of Panax ginseng C.A. Meyer in healthy subjects: a randomized, placebo-controlled clinical trial. Food Chem Toxicol 2011;49:2229-2235.

7 Wong CPF, Bandyopadhyay A, Chen CK: Effects of Panax ginseng supplementation on physiology responses during endurance performance. J Mens Health 2011;8:78-80.

8 Liang YL, Liu Y, Yang JW, Liu CX: Studies on pharmacological activities of cultivated Cordyceps sinensis. Phytother Res 1997;11:237-241. 
Lee et al.: Metastatic Colorectal Cancer Treated with Herbal Pharmacopuncture during FOLFIRI Chemotherapy: A Case Report

9 Dong CH, Yao YJ: In vitro evaluation of antioxidant activities of aqueous extracts from natural and cultured mycelia of Cordyceps sinensis. LWT-Food Sci Technol 2008;41:669-677.

10 Wu JY, Zhang QX, Leung PH: Inhibitory effects of ethyl acetate extract of Cordyceps sinensis mycelium on various cancer cells in culture and B16 melanoma in C57BL/6 mice. Phytomedicine 2007;14:43-49.

11 Korean Institute of Pharmacopuncture: Pharmacopuncturology. Seoul, Elsevier Korea LLC, 2008, pp 2-4.

-12 Han JB, Im CR, Lee JW, Kim SS, Seong S: A case of inoperable klatskin tumor showing response to wild ginseng pharmacopunture. Orient Pharm Exp Med 2013;13:235-237.

-13 Kwon KR, Kim H, Kim JS, Yoo HS, Cho CK: Case series of non-small cell lung cancer treated with wild ginseng pharmacopuncture. J Acupunct Meridian Stud 2011;4:61-68.

14 Donald IA, Andrew TW: Integrative Oncology. Oxford, Oxford University Press, 2009, pp 124-125.

15 Iwase Y, Takemura Y, Ju-ichi M, Mukainaka T, Ichiishi E, Ito C, Furukawa H, Yano M, Tokuda H, Nishino H: Inhibitory effect of flavonoid derivatives on Epstein-Barr virus activation and two-stage carcinogenesis of skin tumors. Cancer Lett 2001;173:105-109.

Table 1. Changes in the severity of symptoms (VAS score) before and after the administration of herbal medicines

\begin{tabular}{lll}
\hline $\begin{array}{l}\text { Chief complaint: traditional } \\
\text { herbal medicine }\end{array}$ & $\begin{array}{l}\text { Date and duration } \\
\text { of medication }\end{array}$ & $\begin{array}{l}\text { VAS } \\
\text { score }\end{array}$ \\
\hline Headache: & $\begin{array}{l}2012 / 10 / 06- \\
\text { Cheong-sang-gyeon-tong-tang }\end{array}$ & 4 \\
& $2012 / 10 / 09$ & 0 \\
\cline { 2 - 3 } & $2012 / 11 / 10-$ & 4 \\
& $2012 / 11 / 24$ & 1 \\
\cline { 2 - 3 } & $2012 / 12 / 01-$ & 4 \\
& $2012 / 12 / 10$ & 2 \\
\hline Nausea: Yi-jin-tang & $2012 / 10 / 09-$ & 6 \\
& $2012 / 10 / 27$ & 1 \\
\hline & $2012 / 11 / 24-$ & 5 \\
& $2012 / 12 / 10$ & 2 \\
\hline Stomatitis: & $2012 / 10 / 18-$ & 6 \\
Hoei-choon-yang-gyeok-san & $2012 / 10 / 27$ & 0 \\
\cline { 2 - 3 } & $2012 / 11 / 24-$ & 4 \\
& $2012 / 12 / 01$ & 3 \\
\hline
\end{tabular}




\section{Case Reports in Oncology}
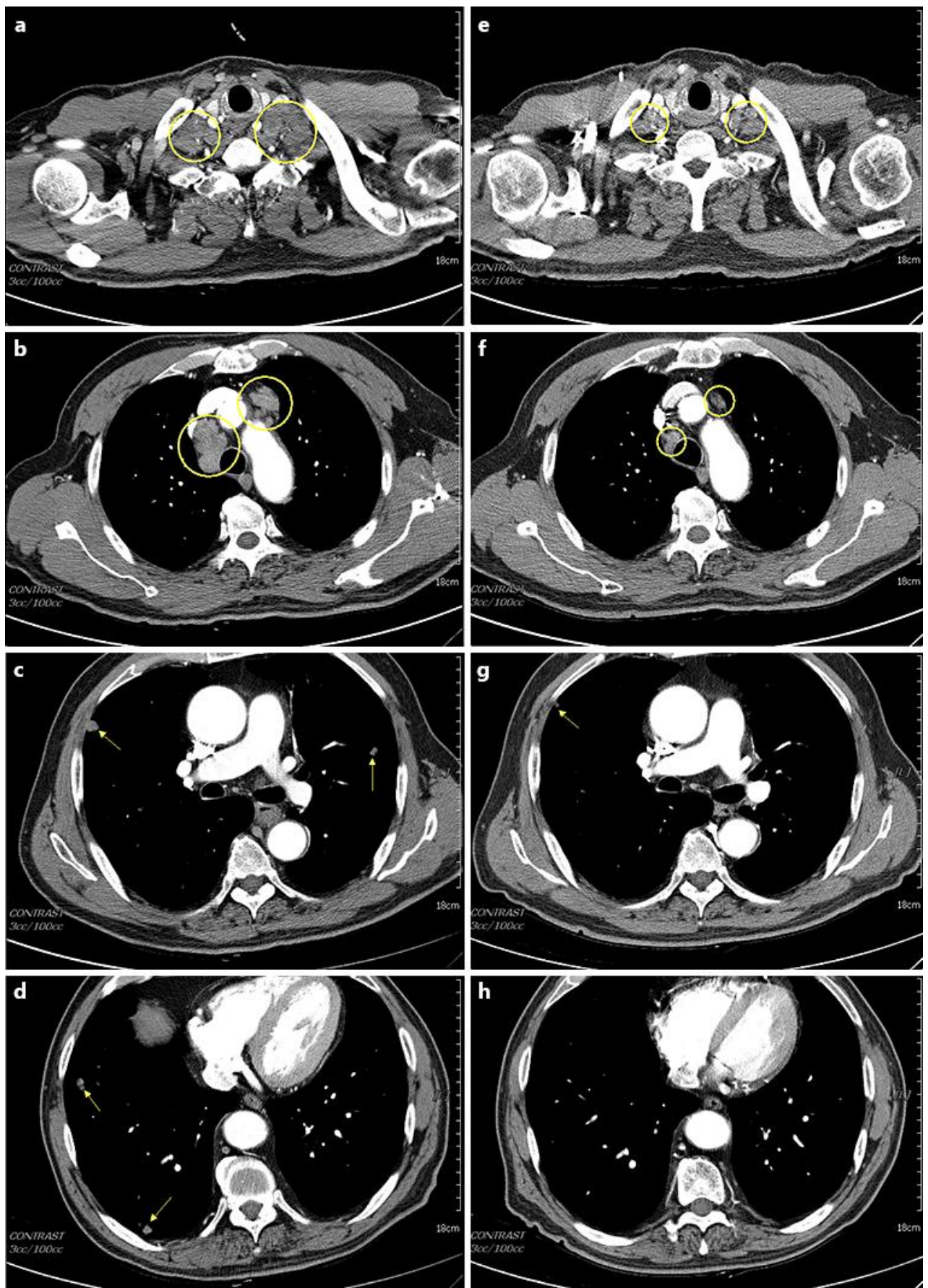

Fig. 1. CT images. Compared to the CT images taken in September 2012 (a-d), the size of the tumors decreased significantly in the CT images of January $2013(\mathbf{e}-\mathbf{h})$, in both the pulmonary and lymph node areas. 